\title{
HispanismeS
}

Revue de la Société des Hispanistes Français

$18 \mid 2021$

Murs, barrières, obstacles dans les mondes hispaniques II

\section{Paco Gómez, un photographe espagnol face au mur à la fin des années 1950}

Paco Gómez, a Spanish photographer facing the wall in the late 1950s

Paco Gómez, un fotógrafo español frente a la pared a finales de los años 1950

Jacques Terrasa

\section{OpenEdition}

Journals

Édition électronique

URL : https://journals.openedition.org/hispanismes/14027

DOI : 10.4000/hispanismes. 14027

ISSN : 2270-0765

Éditeur

Société des Hispanistes Français

\section{Référence électronique}

Jacques Terrasa, «Paco Gómez, un photographe espagnol face au mur à la fin des années 1950 », Hispanismes [En ligne], 18| 2021, mis en ligne le 31 décembre 2021, consulté le 30 janvier 2022. URL : http://journals.openedition.org/hispanismes/14027; DOI : https://doi.org/10.4000/hispanismes. 14027

Ce document a été généré automatiquement le 30 janvier 2022.

\section{cc)}

Les contenus de cette revue sont mis à disposition selon les termes de la Licence Creative Commons Attribution - Pas d'Utilisation Commerciale - Pas de Modification 4.0 International. 


\title{
Paco Gómez, un photographe espagnol face au mur à la fin des années 1950
}

\author{
Paco Gómez, a Spanish photographer facing the wall in the late 1950s \\ Paco Gómez, un fotógrafo español frente a la pared a finales de los años 1950
}

Jacques Terrasa

1 Lorsqu'un photographe se retrouve face au mur, serait-il comme l'écrivain devant sa page blanche ou le peintre face à sa toile : confronté à une surface à remplir? Le plus souvent, les murs qui nous entourent n'offrent qu'une superficie homogène, privée de figures, textes ou autres diversions. L'écrivain remplit sa page de mots; le peintre couvre de taches sa toile - leur rôle étant d'agencer le matériau qu'ils introduisent sur ces surfaces. Mais un photographe - face à un mur blanc- remplira-il son viseur comme un peintre barbouille sa toile ? La réponse est négative, car si le photographe agence aussi des formes, il ne le fait pas en accumulant des mots ou des coups de pinceau qu'il introduit sur le vide du support, mais au contraire, en partant du tropplein de motifs, de lumière et de couleurs que lui offre déjà ce monde extérieur, fût-il un mur recouvert de taches et de graffitis. Son rôle est alors de le vider, de laisser hors champ ou dans la pénombre tout ce que le réel a d'excessif, pour n'en garder que deux ou trois petites choses qui, savamment placées dans la lumière, donneront la bonne photo.

2 Le paradoxe du photographe de murs, c'est qu'il travaille sur une surface plane, comme un peintre ou un écrivain, et non sur l'agencement des volumes dans la lumière, comme le font ses collègues qui, au lieu de se planter comme lui devant l'écran opaque du mur, acceptent les trois dimensions du monde. En tournant le dos à cette pratique courante, le photographe de murs se prive de ce qui constitue la photographie depuis deux siècles : organiser à l'intérieur de la camera obscura, selon les lois de la perspective, les faisceaux lumineux qui se sont réfléchis sur l'espace complexe du monde tridimensionnel, pour aller ensuite s'écraser durant une fraction de seconde sur la surface plane de la pellicule argentique. En photographiant un mur de manière 
frontale, comme le fait Paco Gómez (dont il va être question ici), celui-ci se limite-t-il à faire coïncider deux surfaces planes? On sait que certains pionniers de la photographie, comme William Henry Fox Talbot, enregistraient au milieu du XIX ${ }^{e}$ siècle l'empreinte lumineuse des feuilles et des fleurs, en posant celles-ci directement sur le support sensible. À l'instar d'un graveur recueillant l'empreinte de la planche sur le papier, Talbot recueillait dans ses photogrammes celle de la nature directement enregistrée par le papier photographique, sans l'intermédiaire d'une chambre. Mais les images dont il va être question ne sont pas ces photogrammes d'un autre siècle, mais bien de classiques images prises au Rolleiflex par quelqu'un qui renonce, du moins dans une partie de son œuvre, à mettre à plat les volumes et la profondeur du monde extérieur, pour n'en saisir que des formes déjà planes à l'origine. Étrange attitude, peu fréquente dans le monde de la photographie, à l'exception d'artistes comme Brassaï, dont les clichés de graffiti, réalisés dès le début des années 1930, avaient été exposés au MoMA en 1956. Nous verrons que les photos de Paco Gómez ont peu de rapport avec celles de Brassaï, lequel, dans un esprit surréaliste, choisissait d'exacerber les détails graphiques fonctionnant comme des objets trouvés sur les murs de Paris. Pour Gómez, c'est l'objet-mur qui importe, même s'il est parfois isolé par le cadrage, comme chez Brassaï, comme on le voit, par exemple, sur deux photos Sin título, l'une de 1958, avec le dessin d'un oiseau et l'inscription «Misión ", et l'autre prise vers 1960, où l'on voit le graffiti d'une tête ronde. Mais en fait, les «murs» de Gómez sont le plus souvent contextualisés.

Reprenons l'histoire par son début, c'est-à-dire en 1956, l'année où Brassaï expose à New York. Cette année-là, dans un coin reculé d'Andalousie, deux photographes, José María Artero et Carlos Pérez Siquier, créent AFAL, «Asociación Fotográfica Almeriense ", et publient dans la foulée, et ce jusqu'en 1963, la revue photographique de même nom. AFAL édite même, en 1958, un précieux Anuario de la Fotografía Española qui contribuera amplement à la connaissance de celle-ci ${ }^{1}$. Comme cela est signalé dans le Lexique bilingue des arts visuels, "AFAL a marqué une nouvelle orientation dans la photographie espagnole, consacrée jusque-là aux reportages de propagande ou au pictorialisme tardif, réalisés dans le contexte très surveillé des clubs et des concours photo $»^{2}$. Francisco Gómez Martínez (Pamplona, 1918-Madrid, 1998), ainsi que son ami Gabriel Cualladó (1925-2003), un autre photographe, appartenant comme lui à la Real Sociedad Fotográfica de Madrid, sont devenus membres d'AFAL dès 1957; Gómez et Cualladó ont participé cette année-là à une exposition collective dans la capitale, avant d'inaugurer l'année suivante la première d'une série d'expositions en tandem, qu'ils poursuivront durant toute leur carrière. Pour Gómez (mais on pourrait dire la même chose de Cualladó) :

La entrada en AFAL impulsa radicalmente su evolución, especialmente por el contexto nuevo en el que se sitúa: nuevas iniciativas y planteamientos, intercambios, actualización, proyección, conexión con otros grupos fotográficos europeos, y especialmente exposiciones que le obligarán a definir su propuesta ${ }^{3}$.

Soyons clairs: Cualladó n'est pas un photographe de murs; l'être humain, mis en situation au long des années dures du franquisme, est essentiel dans ses photos. Toutefois, dans certaines d'entre elles, le mur fait part égale avec l'humain. C'est le cas d'une de ses images les plus connues, où un mur, vu en vision frontale et occupant une place prépondérante dans la composition, surmonte la figure d'une vieille dame, assise sur un banc et entourée de bagages, dans la photographie en noir et blanc intitulée Sala de espera en la estación de Atocha, Madrid, 1957. J'ai déjà, au siècle dernier, analysé ce 
document dans un ouvrage méthodologique régulièrement réédité depuis; je l'avais choisie comme exemple de ce que la photographie espagnole avait produit de meilleur sous le franquisme ${ }^{4}$. Je ne reprendrai pas ici mon commentaire d'il y a 22 ans. Ce qui m'intéresse aujourd'hui, c'est comprendre mon choix : celui d'une image où « les $9 / 10^{\text {e }}$ de la surface renvoient à des zones planes, parallèles au plan de l'appareil photo (mur, dossier du banc, bagages...) »5. En quelque sorte, une image où la tridimensionnalité du monde extérieur est neutralisée, pour privilégier l'agencement des taches et des valeurs de gris, comme l'avaient fait les peintres abstraits au début du $\mathrm{xx}^{\mathrm{e}}$ siècle, lorsqu'ils ont décidé de tourner le dos à l'illusion de profondeur pour privilégier l'agencement des formes et des couleurs dans une toile devenue autonome. Pourquoi certains photographes espagnols - comme Gabriel Cualladó ici, mais surtout Paco Gómez, qui va nous occuper dans tout cet article - neutralisent l'illusion de profondeur pour s'intéresser aux textures ? Je suggérais déjà la piste de la peinture informelle à l'époque, avant de proposer une interprétation spéculaire, dans les dernières lignes de mon commentaire :

Il n'y a pas, comme dans Les Ménines, de miroir dans cette salle d'attente de la gare d'Atocha ; pourtant, les sels d'argent qui composent cette représentation du grand mur gris créent ici une surface spéculaire où se reflètent nos propres doutes, une surface où Gabriel Cualladó projette l'espace intérieur de la vieille dame, tandis que le portraitiste, en bas, reste prisonnier de l'apparence des choses $^{6}$.

\section{« La Palangana »}

5 On vient de voir que certains photographes de la génération des années cinquantesoixante ont recherché dans les murs le cadre idéal pour les portraits en situation qu'ils nous ont livrés des Espagnols plongés depuis bientôt un quart de siècle dans une dictature honnie - et les injonctions du Régime pour leur faire croire qu'ils avaient vécu, comme l'a proclamé Franco en 1964, « 25 años de paz», ne les ont pas fait changer d'opinion! Pourtant, seul Paco Gómez a été ce que l'on peut considérer comme « un photographe de murs ». Ses amis les plus proches - ceux qui gardent l'être humain au centre de leurs photographies - s'appellent Ramón Masats, Francisco Ontañón, Leonardo Cantero, Joaquín Rubio Camín, et bien sûr Gabriel Cualladó. Ces six photographes se retrouvaient souvent pour dîner à la Cervecería Alemana, sur la madrilène place Santa Ana, où ils discutaient ensuite jusqu'à tard dans la nuit. C'est là qu'ils ont décidé de créer en 1959 un groupe relativement informel, que Masats a baptisé «La Palangana », en référence aux cuvettes que les photographes emploient dans leur $\mathrm{labo}^{7}$; Ontañón immortalisera le groupe en prenant le cliché d'une cuvette en fer émaillé, pleine d'eau et posée sur une chaise, où flottent les six photos des membres du groupe ${ }^{8}$. La Palangana, désignée par Laura Terré comme «le groupe madrilène d'AFAL ", aura une vie éphémère, puisque dès le début des années soixante, les photographes madrilènes associés à AFAL ont été désignés par l'expression «École de Madrid». 
Francisco Gómez, Cristo, 1958-59.

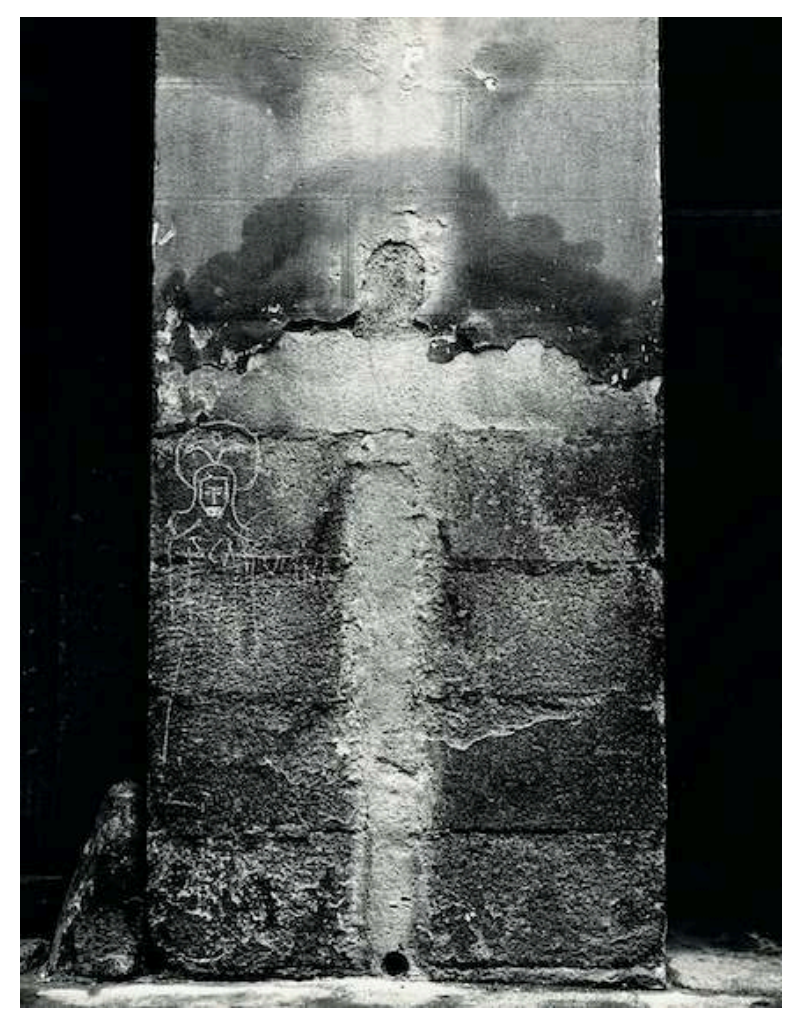

(C) Archivo Paco Gómez / Fundación Foto Colectania

6 Se démarquant en cela des autres membres du groupe, Paco Gómez recherche surtout les traces, les ruines, les formes inanimées qui, même si elles gardent la mémoire de l'humain, donnent à travers son viseur des compositions frontales, statiques, souvent aux limites de l'abstraction. Si nous feuilletons l'ouvrage Paco Gómez. Fotografías, publié en 2010 à l'occasion de l'exposition « Paco Gómez. Orden y desorden ", sous la houlette de Laura Ferré, nous constatons que plus de $40 \%$ des 73 photos sélectionnées pour l'ouvrage sont des images de murs ${ }^{10}$. Cependant, Gómez a surtout été connu, entre 1959 et 1974, pour ses quinze années de collaboration intensive à la revue Arquitectura. C'est son directeur, Carlos de Miguel, qui avait fait appel à lui en 1959, comme il le raconte dans le numéro 169-170, daté de janvier-février 1973, au moment de son départ à la retraite, après 25 années à la tête de la revue :

Se trata de un fotógrafo aficionado que me presentó su paisano el arquitecto Rafael Alfonso Corral. Ha sido un sensacional fichaje para la revista, porque se ha especializado en temas de arquitectura, con el trato de diferentes arquitectos, y ahora [...] es un auténtico as en ese difícil menester ${ }^{11}$.

Photographe amateur, Paco Gómez l'a été toute sa vie, comme la plupart de ses collègues - car en dehors des photographes de presse, de quelques rares publicitaires ou photographes de mode, ou de ceux qui pouvaient ouvrir une enseigne commerciale de portraits et reportages, il fallait bien posséder à côté une activité plus lucrative. Pour Gómez, diplômé d'une école de commerce, c'est son travail dans le magasin de confection de son oncle qui lui a permis de disposer de l'aisance financière nécessaire pour pratiquer la photographie dès 1943. Son ami Ramón Masats, arrivé à Madrid en 1957 pour travailler comme professionnel à La Gaceta Ilustrada, se souvient de cette époque dans sa courte introduction au volume que la collection PHotoBolsillo a 
consacré à Paco Gómez : « Por entonces, Paco trabajaba en la sastrería de su tío rico en el centro de Madrid, en la Red de San Luis, esquina con Gran Vía. El tío era soltero y Paco su heredero ${ }^{12}$. "Si à la fin de 1957, Gómez participe, comme on l'a vu, à une première exposition collective, c'est à partir de l'année suivante qu'il réalise les photos qui figureront en décembre 1959 dans une exposition de plusieurs membres du groupe AFAL à Paris, puis en janvier 1960 dans la Sala Darro, à Madrid, avec Gabriel Cualladó ${ }^{13}$; certaines de ces photos se trouvaient déjà quelques mois plus tôt dans le portfolio qu'il avait montré à son ami architecte navarrais ${ }^{14}$, et qui, par l'entremise de celui-ci, lui ont donné accès à la revue Arquitectura. Mais de quelles images s'agissait-il ?

Ces photos de 1958-59, où la présence des murs est importante, ont été prises lors de ses sorties avec ses amis photographes, dans différents villages et petites villes de Castilla-La Vieja, comme on disait à l'époque. Mais la plupart proviennent de la périphérie de Madrid, des zones limite de certains quartiers, où il se rend avec son Rolleiflex, appareil de moyen format dont les négatifs de $6 \times 6 \mathrm{~cm}$ permettent ensuite les recadrages nécessaires ${ }^{15}$, à l'inverse de ce qui se passe généralement avec les négatifs $24 \times 36 \mathrm{~mm}$. "Los domingos por la mañana me iba a hacer fotos. La tarde positivando y a las tres de la mañana esmaltando ${ }^{16}$, a-t-il dit à Laura Terré, lors d'un entretien qu'elle a enregistré à Almería en 1991, à l'occasion de l'exposition «AFAL 1956-1991 ». Son ami Ramón Masats se souvient de ces sorties dominicales des six membres de La Palangana :

Algunos domingos por la mañana nos juntábamos en el recién construido Barrio de la Concepción, donde vivía Rubio Camín, casado y con dos hijas. Tomábamos café en un bareto y luego nos dispersábamos, cada uno a lo suyo. Leica y Rolleiflex. Fotografiábamos los descampados aledaños y sus gentes, el Arroyo del Abroñigal (hoy M-30), sus viejas casas... A una hora convenida, todos volvíamos al mismo bar. Cervecita, anécdotas pertinentes y comentarios fotográficos. Luego a casa, donde la mujer, los que la tenían, les había preparado la suculenta ${ }^{17}$.

9 En vérité, les images contemplatives de Gómez requerraient une solitude et une lumière, parfois incompatibles avec des sorties programmées. Aussi, pratiquait-il souvent des repérages, avant de revenir sur les lieux dans de bonnes conditions de luminosité, comme l'explique Rafael Levenfeld:

Su mirada pausada siempre estuvo atenta a los códigos secretos de la ciudad ocultada. En numerosas ocasiones primero fotografiaba mentalmente, sin la cámara. Realizaba sus paseos a distintas horas del día para ver cómo la luz iba transformando los volúmenes. A menudo él decía: «Las paredes siempre me esperan ». Después volvía con su Rolleiflex a la hora precisa y tomaba la fotografía definitiva ${ }^{18}$.

De toute cette pratique, lente et régulière, se dégagent une esthétique et une éthique de la photographie qui lui sont propres. "Los movidos, los barridos, todas esas cosas modernas... no son para mí. Lo mío es una fotografía más purista. Hay cosas que yo no haré nunca, como introducir desenfoques o poner filtros para modificar la luz, el color o el contraste... ", disait-i ${ }^{19}$. On trouve dans ses photos très peu de présence humaine effective, comme cela a déjà été dit ; mais par contre, on y ressent toujours la trace de l'Homme, ainsi que la présence du temps, de l'Histoire qui s'inscrit sur les murs, dans les brèches. Alberto Martín énumère, dans une longue phrase, les caractéristiques de la pratique photographique de Francisco Gómez :

Se destaca en él el uso de la frontalidad, el estatismo, la austeridad, el efecto de los contrastes, la esquematización de la materia y de los objetos, el grafismo, la profundización en las regiones más íntimas de lo inanimado, su atención al paisaje, 
el acto de desdoblar lo visto y lo presentido, el valor poético, la meticulosidad, sensibilidad e instinto de observación, y, especialmente, su subjetividad artística, el modo en que el espíritu del realizador está inequivocadamente en el fondo de sus obras $^{20}$.

\section{L'austère frontalité des photographies de murs}

Francisco Gómez, Butaquita en un frontón, 1957.

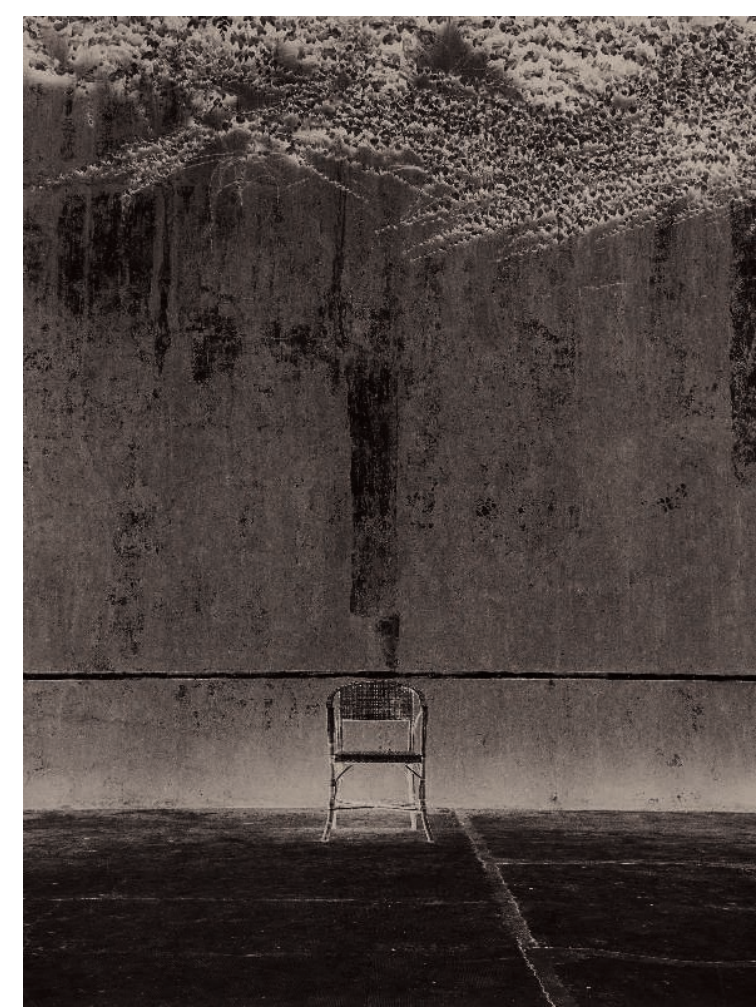

(C) Archivo Paco Gómez / Fundación Foto Colectania

11 L'une de ses photos les plus connues de 1958-1959 s'intitule Cristo; le titre est anecdotique, dans la lignée des concours que les associations photographiques organisaient en Espagne dans les années cinquante. Sur un mur décrépi et couvert de taches d'humidité, une bande verticale, centrée et plus claire que son pourtour, s'élevant aux deux tiers de l'image, croise une bande horizontale qui divise harmonieusement la photographie dans un rapport proche du nombre d'or. L'ensemble forme une croix qui, avec le graffiti bien visible, à gauche, aux allures d'un saint barbu et auréolé, renforce les connotations christiques. Mais ce titre est une exception : les photos de Paco Gómez sont généralement référencées Sin título, ou alors avec une indication de lieu plus précise que le simple nom de la ville (Alrededores de la avenida de América) ou de l'objet (Huellas; Bodegón con ladrillo). Aussi, le choix de Cristo pour ouvrir ces commentaires n'a rien à voir avec l'allusion religieuse, mais vient du fait que c'est à son sujet que Matías Ballester Cairat, dans un article qu'il publie en 1961 dans Arte fotográfico $^{21}$, a employé pour la première fois le terme informal à propos d'une de ses photos $^{22}$. Avec le recul des ans et la connaissance que l'on a aujourd'hui de l'œuvre d'Antoni Tàpies, il semble évident que certaines images de murs de Gómez entrent en consonance avec certains tableaux du plus connu des peintres informalistas, que celui-ci 
peint exactement à la même époque dans son atelier barcelonais. En effet, si l'on observe les tableaux réalisés avec un mélange de latex et de poudre de marbre en 1958 ou $1959^{23}$, la contemplation silencieuse à laquelle Antoni Tàpies nous invite, face à ce qu'il désigne lui-même comme ses « murs ${ }^{24}$, n'est pas éloignée de ce que nous révèlent les photographies de murs de Paco Gómez : une poétique des empreintes, des griffures et des graffitis, une lente érosion de la matière, les taches mystérieuses que le temps trace sur les murs décrépis... On y retrouve bien la "poétique de la matière " propre au peintre catalan - l'expression est d'ailleurs de Saramaya Pelletey, qui en a fait le titre de sa thèse : Un artiste face au mur : Antoni Tàpies et la poétique de la matière (1955-1975) ${ }^{25}$. La "charge métaphysique ${ }^{26}$ que possèdent les photos de murs de Paco Gómez - que Carlos de Miguel choisit pour les couvertures ou pour accompagner des articles de fond de la revue Arquitectura-, contribue au fait que ces images, selon Alberto Martín, dialoguent directement avec l'informalisme ${ }^{27}$. Nous sommes loin des problématiques de la photographie amateur de l'époque, avec ces œuvres qui nous rapprochent d'une abstraction aux connotations picturales.

Aquí, el continente, la medianería o el muro han dejado paso al contenido, ya no hay reconocimiento físico posible del contexto donde se localiza, la humedad ahora es mancha, y las marcas, incisiones o rugosidades son texturas. Lo cierto es que Gómez preserva este contenido más explícitamente abstracto, conectado directamente con el informalismo, para las páginas de la revista, alejado del campo fotográfico, en el que quizás no se viera nada más que una coincidencia de resultados con respecto a la pintura ${ }^{28}$.

Francisco Gómez, Horizontal y vertical, 1960.

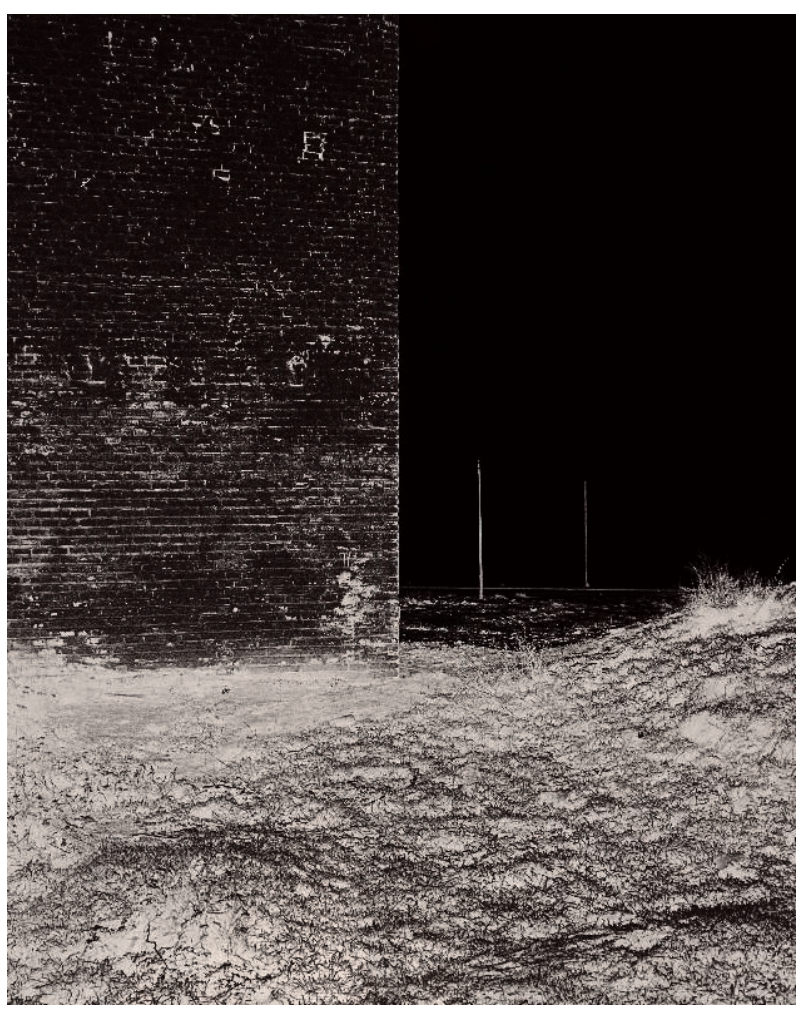

(c) Archivo Paco Gómez / Fundación Foto Colectania

12 Mais si l'on fait exception des quelques photographies qui rappellent Brassaï, on ne saurait réduire ces photographies de murs à de simples rectangles découpés - comme 
au cutter - sur une surface plane. Si c'était le cas, la neutralisation des lois de la perspective, pour ne conserver de la troisième dimension que les effets de texture, reviendrait à une pratique similaire à la reproduction de tableaux. Cette manière de procéder, qui se rapproche du ready made, ou de l'« objet trouvé ", pour lequel l'artistephotographe limite son rôle au maximum, convient pour le travail d'un surréaliste comme Brassaï : l'image préexiste à l'instant de sa prise de vue; il suffit de la voir, et de choisir la lumière. Cette pratique minimaliste n'est cependant pas évidente, et on en trouve malgré tout des beaux exemples dans l'œuvre de Paco Gómez. Laura Terré, dans son choix pour le livre de 2010, en a sélectionné quelques-unes. Citons par exemple Tapia de Cáceres (1961), une image totalement abstraite, aux purs effets de craquelures et de moisissures. La figure aux contours organiques pourrait avoir des connotations anthropomorphiques lorsqu'elle est disposée verticalement ; mais telle qu'elle apparaît, imprimée à l'horizontale sur la couverture du no 58 (octobre 1963) de Arquitectura, les territoires étranges formés par les taches évoquent alors quelques organismes monocellulaires ou un paysage lunaire. Mochuelo y pared en la carretera (1962) est plus simple: une forme blanche et craquelée sur fond sombre, avec dans le haut, un rectangle horizontal, allongé, percé de deux orifices - empreinte probablement laissée par une planchette arrachée - crée l'illusion, celle d'un mochuelo qui nous observe. Le plaisir de voir surgir une chouette blanche au milieu du mur conforte notre prédisposition à repérer dans des taches abstraites des formes signifiantes. Personne n'est dupe, mais le jeu popularisé par Léonard de Vinci fonctionne toujours.

13 La photographie Cristo, point de départ de ces réflexions, est toutefois différente de Tapia... et Mochuelo..., pour lesquels seule la texture du mur renvoie à une troisième dimension spatiale. En effet, de chaque côté de la paroi marquée d'une croix grise, on a deux passages obscurs vers des espaces latéraux, perpendiculaires au mur. On s'enfonce dans le noir, là où le bout de trottoir visible dans le bas de l'image se prolonge des deux côtés, avec à gauche, une pierre triangulaire posée contre le mur, et dans l'angle inférieur droit, le dallage qui s'enfonce dans le noir. Les deux bandes obscures, que l'on pourrait confondre avec le passe-partout de l'image encadrée ou la page noire qui entoure l'image imprimée, occupent un quart de la surface, et révèlent une troisième dimension qui contredit la frontalité de l'image. Enfin, le conduit d'évacuation des eaux pluviales qui débouche en bas, sur le trottoir, provient selon toute vraisemblance de la saignée verticale, dans le mur, remplie de ciment gris. Ainsi décrite, l'image a perdu son mystère, en retrouvant une partie de son contexte urbain et architectural. Mais Gómez ne recherche pas dans un cadrage serré l'étrangeté surréaliste chère à Brassaï. Il appartient à une génération marquée par le néoréalisme et la photographie humaniste, et pour lui, le mur dialogue avec son environnement, car c'est ce dialogue qui fera la force des images. On va le voir dans les trois dernières images de Paco Gómez que j'ai choisi de commenter. Toutes datent de la même période, comprise entre 1957 et 1962. C'est alors qu'il réalise ses meilleures photos - même si, durant les deux décennies suivantes, il nous livrera de superbes images, avec même une incursion dans le domaine de la photographie couleur durant les années 1980. 
Francisco Gómez, Tapia en la calle Toledo, 1961.

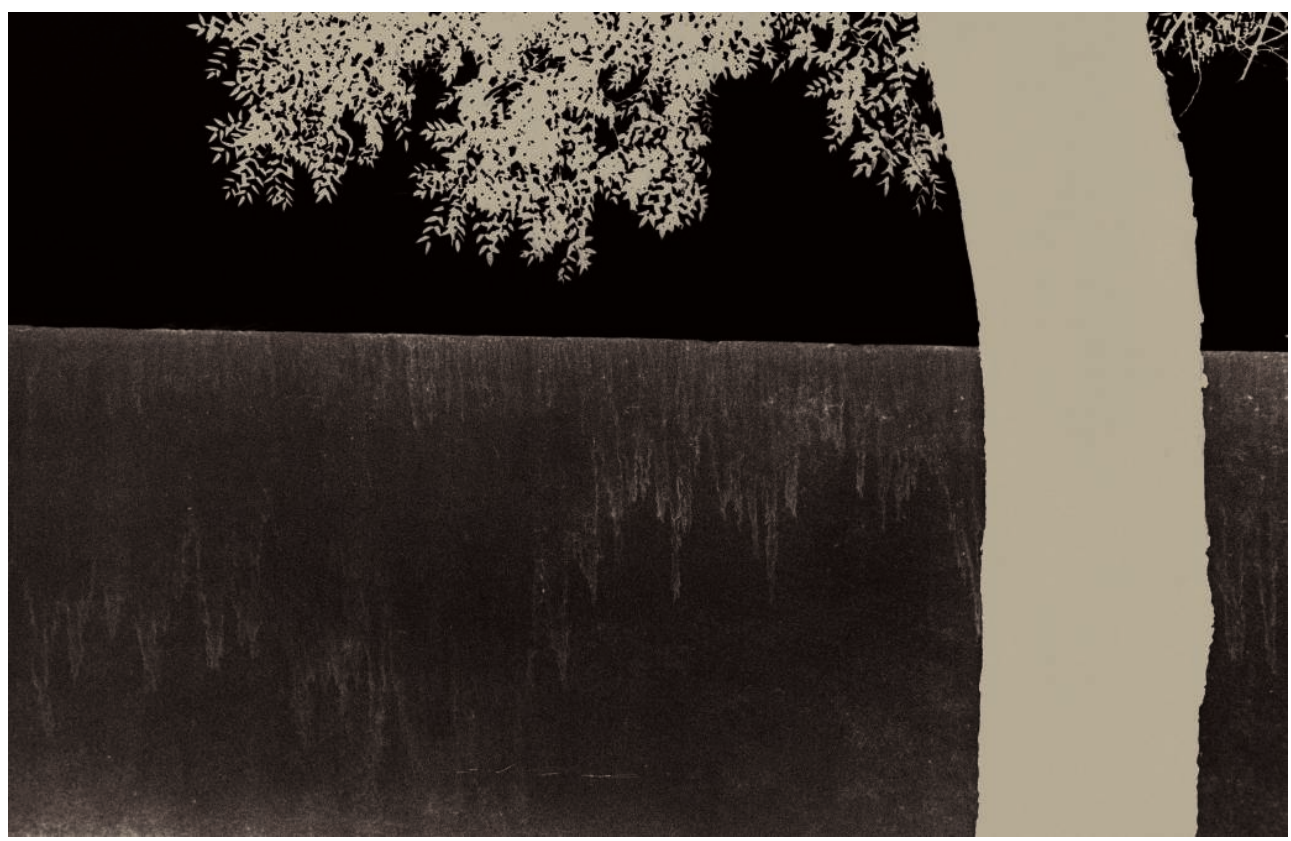

(c) Archivo Paco Gómez / Fundación Foto Colectania Rolleiflex; le tirage d'époque conservé mesure en effet $55 \times 50 \mathrm{~cm}$. La frontalité du cadrage est en consonance avec le titre choisi, un fronton de pelote basque contre lequel s'appuie un fauteuil en osier, parfaitement centré au-dessous d'une ligne horizontale blanche, placée à la limite du quart inférieur de l'image. Dans le quart supérieur, un lierre aux contours organiques court le long du fronton. Butaquita... faisait partie du choix de dix photos que Paco Gómez a présentées à Paris en décembre 1959 pour l'exposition commune des membres d'AFAL et du groupe français Les $30 \mathrm{x}$ $40^{29}$. On y trouve la rigueur dans les cadrages et le soin qu'il apportait au travail de la lumière, mais aussi la liberté qu'éveille la distribution aléatoire des taches - deux caractéristiques de ses photos de murs. Le fronton est ici comme un miroir, qui ne nous renvoie plus la pelote, mais notre propre image contemplative. Cependant, à l'inverse de la photo de Cualladó datée de la même année, aucune vieille dame n'est assise au bas de l'image, et le fragile fauteuil en osier ne nous invite qu'au repos et à l'observation. Son occupant est parti, nous cédant sa place. L'image est réversible; on nous imagine facilement dans le contrechamp, prenant le rôle de l'observateur paisible méditant face à l'impermanence des choses, observant la patine du temps sur ce mur décrépi, sensible à la beauté éphémère et imparfaite du monde, conscient de sa propre finitude ${ }^{30} \ldots \mathrm{Au}-$ dessus de la ligne blanche, c'est le territoire presque bidimensionnel des textures, minérales et végétales, qui dessinent un paysage abstrait ; au-dessous, c'est l'espace en trois dimensions, avec le fauteuil et les dalles de pierre dans le prolongement desquelles nous nous trouvons. De leur interaction nait l'image.

La photographie de 1960 intitulée Horizontal y vertical est conforme à son titre : une ligne verticale sépare, dans les deux-tiers supérieurs de l'image, deux rectangles d'une superficie identique, qui opposent le vide et le plein. On a, en effet, dans la moitié gauche, un grand mur aveugle, avec une trame dense de briques chaulées, et dans la moitié droite, une zone d'un gris très clair, où seuls deux poteaux électriques 
emplissent avec délicatesse le vide du ciel. Enfin, la terre et les mauvaises herbes du tiers inférieur composent dans la lumière rasante une symphonie de gris sombres où se révèle tout le talent du tireur. À droite de cette zone, un petit tertre buissonneux rompt une géométrie trop parfaite, et rééquilibre l'image vers la partie où se trouve le punctum de la composition: les deux poteaux, ces deux traits fins servant de contrepoint à la pesanteur des masses. Paco Gómez était un photographe du sensible, tout l'opposé d'un conceptuel. Ses compositions rigoureuses cachent toujours le détail fragile qui suscite l'émotion.

Notre dernier commentaire portera sur une photo presque abstraite, intitulée Tapia en la calle Toledo, Madrid, 1961. Comme pour la précédente, Gómez juxtapose trois valeurs : l'une, très foncée, pour un tronc d'arbre (à droite de l'image) et son feuillage (quelques branches qui font penser aux feuilles d'un faux-poivrier, occupent le haut de l'image) ; la seconde valeur, d'un gris moyen, est celle d'un muret, souillé par des coulures probablement dues aux intempéries; enfin, au-dessus de la tapia, un autre rectangle, parfaitement blanc, semble correspondre à un mur chaulé ou un ciel dégagé, à l'arrièreplan. C'est austère, schématique, statique et contrasté... pour reprendre des termes d'Alberto Martín. C'est surtout minimaliste. Si prendre une photo consiste à vider le champ visuel de tout ce qui l'encombre pour ne garder que l'essentiel, Paco Gómez touche ici à la perfection! Ce n'est pas un "carré noir sur fond blanc", comme pour Malevitch, mais un " tronc noir sur fond gris ». Cependant, il est difficile de désigner comme un « fond » ce qui fonctionne plutôt comme une " forme » : le muret est surtout perçu comme un long rectangle gris, rythmé par les coulures verticales, délicates comme un lavis; et le tronc noir est d'abord une brèche sombre ouverte dans ce mur, verticale et inquiétante car on ne devine rien de son écorce. Cette brèche, et le blanc parfait de la partie supérieure (où se découpe le tracé compliqué du feuillage), sont les deux extrêmes entre lesquels surgira l'image médiane du mur. Pour une photographie argentique, le travail en laboratoire est essentiel, avec le choix du cadre, le temps d'exposition (qui peut varier en fonction des caches utilisés pour masquer certaines zones) et le contraste choisi pour le papier. Pour Paco Gómez, sa photo n'existe qu'après ce travail de laboratoire. Dans Tapia en la calle Toledo, le recadrage dans l'agrandisseur conduit à un fort travail de coupe. Le feuillage reste largement hors champ ; l'arbre est doublement sectionné par les bords de l'image ; le mur est un ruban dont on ne voit pas la base, et qui se prolonge dans les hors-champs latéraux. 
Francisco Gómez, Tapia en la calle Toledo, con Marichu, 1961.

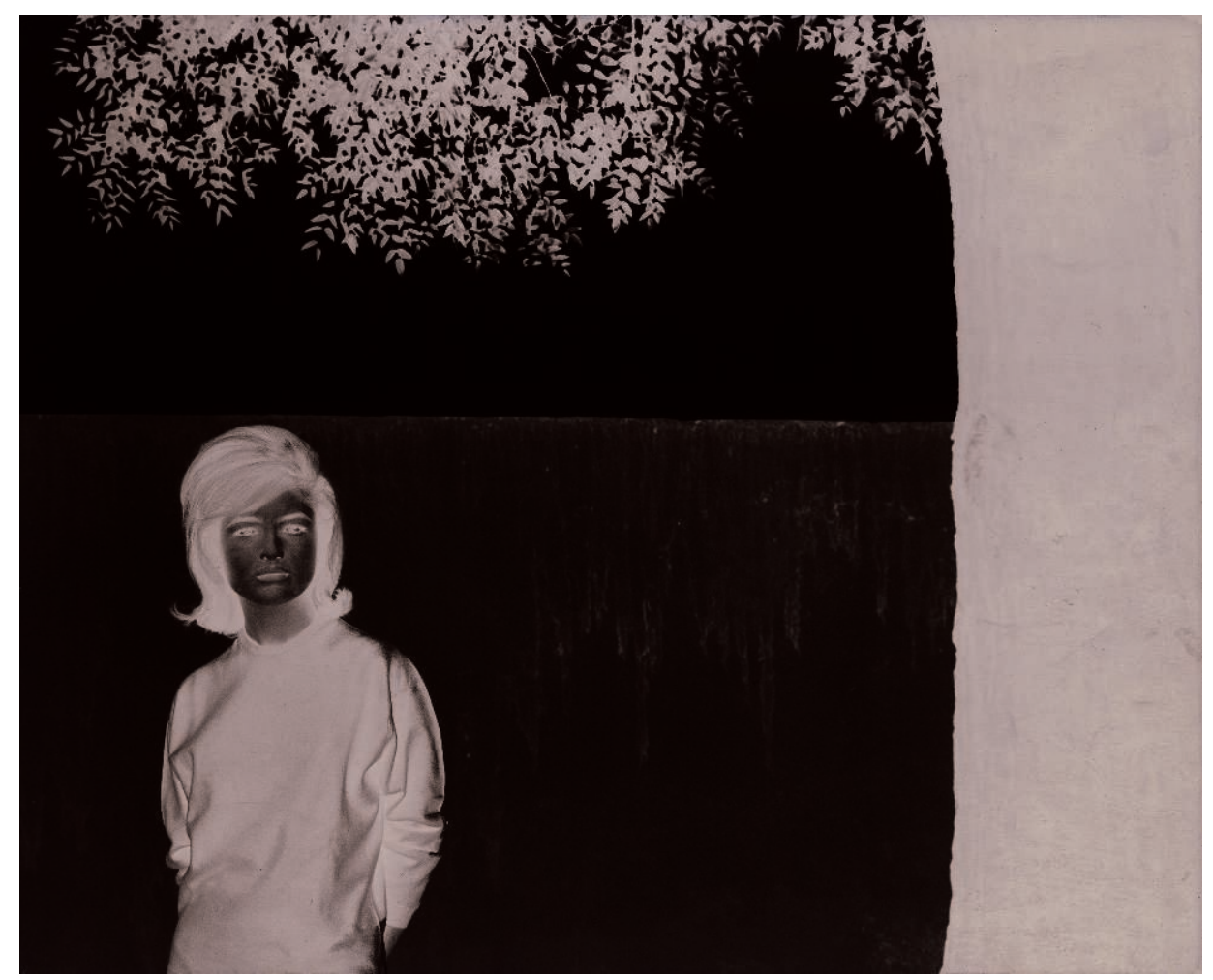

(c) Archivo Paco Gómez / Fundación Foto Colectania

17 Cependant, il suffit que passe une jeune fille, et tout est chamboulé. C'est ce que l'on constate dans une autre photo, prise certainement le même jour, et intitulée Tapia en la calle Toledo, con Marichu. On y voit une jolie brune, l'air farouche, qui pose devant le mur. Elle est dans la partie gauche; de l'autre côté, la partie droite de l'arbre se trouve maintenant hors champ ; enfin, l'image est au format $4 \times 5$, alors que la version sans la jeune fille est au format $2 \times 3$. Ces deux versions ${ }^{31}$ de ce qui semble être une même photo, résument bien la dialectique fond/forme qui sous-tend les quelques photos de murs que nous avons commentées.

\section{Conclusion}

18 À partir des quelques exemples précédents, quel statut a donc le mur dans l'œuvre de Paco Gómez? Si l'on prend ces images dans l'ordre chronologique, on constate que le fauteuil vide de Butaquita en un frontón n'est qu'une forme qui a perdu sa fonction ; c'est la métonymie d'un personnage absent, un siège parfaitement centré au bas du fronton, mais devenu dérisoire. De plus, le diminutif -ita le rend attachant, mais lui enlève toute superbe, car quel prince oserait s'asseoir sur ce trône devenu dangereux, sous ce mur destiné à recevoir des balles ? Cette contamination de la forme par le mur du fond, rongé par le lierre et l'humidité, a fait son œuvre, connotant la durée, l'attente, la morsure du Temps. L'occupant du siège est à présent invisible; il s'est effacé pour que les polarités s'inversent, pour que le mur impose sa sombre présence.

19 C'est là une image qui m'a fait penser dès le départ à deux photomontages de Joan Fontcuberta, datés de l'époque de la Transition. Franco est mort quelques mois plus tôt. 
Sur la première image, intitulée Van declarar frenèticament que només els unia una forta amistat, deux chiens copulent sur un trottoir, devant un mur où l'on peut lire Amnistía total. Cependant, on constate que la chienne est devenue invisible, ne laissant deviner que son contour, tandis que le mâle, un beau dalmatien plein de vigueur, est encore bien présent. Le processus a été enclenché ; l'effacement des formes va se poursuivre, et bien vite, il ne restera que les traces de cette amnistie réclamée en 1976 par les Catalans, à travers les graffitis des murs qui gardent leur parole. Mais c'est l'autre photomontage, dont le titre est Va ser difícil poder dir: ja sóc aquí, qui m'a surtout fait penser à Butaquita... L'image met en scène un cycliste, statique et concentré sur un vélo aux roues à moitié dégonflées. Il s'agrippe au guidon, posant devant un mur couvert de lierre et de vigne vierge. Comme pour la photo de Paco Gómez, les plantes grimpantes ont rongé peu à peu le mur, et peut-être même le personnage également immobilisé, comme par osmose. En 1976, une nouvelle génération de photographes est apparue, mêlant contestation politique et influences fantastiques. Émule de L'Homme invisible de H. G. Wells, le cycliste s'efface peu à peu, et ses jambes sont déjà devenues translucides ${ }^{32}$. "Il n'en fallait pas davantage pour nier le mouvement, l'ouverture spatiale, la liberté habituellement associée à ce sport et tisser une série de connotations autour de l'immobilisme, de l'enfermement, de l'impuissance ${ }^{33}$. » Par d'autres voies que celles empruntées par Gómez, ces deux photomontages montrent que l'instant décisif n'existe pas pour certains photographes, et que les figures du présent s'effacent devant la pérennité des murs.

Joan Fontcuberta, Van declarar frenèticament que només els unia una forta amistat, 1976.

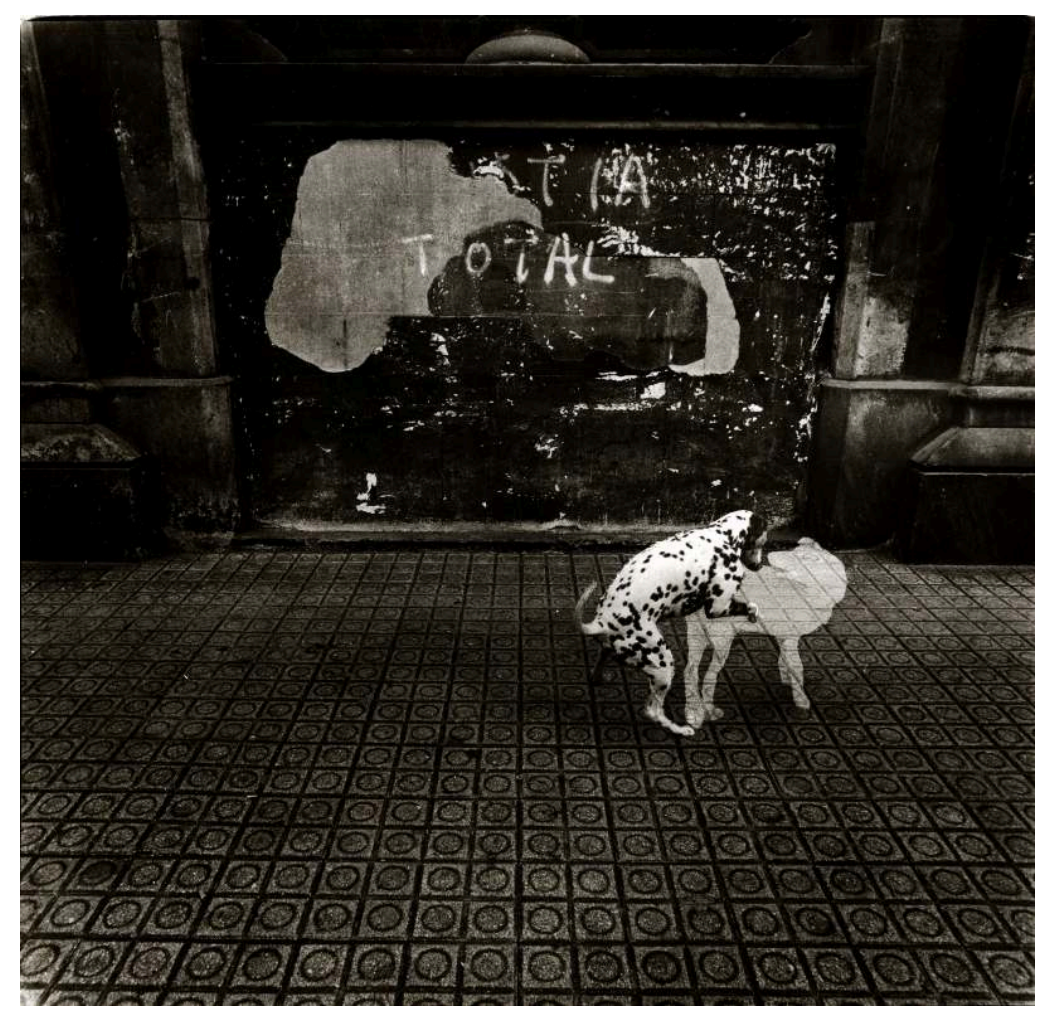

Cortesía Joan Fontcuberta 
Joan Fontcuberta, Va ser difícil poder dir. ja sóc aquí, 1976.

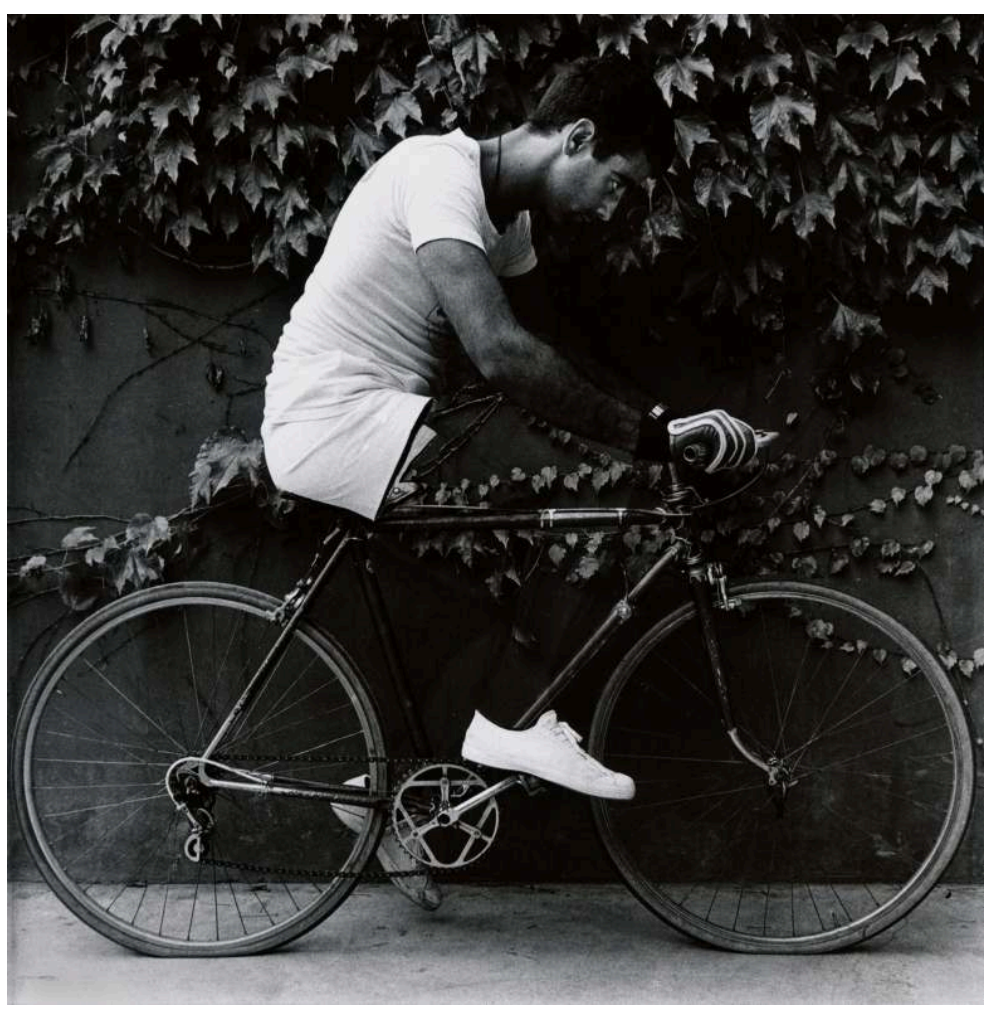

Cortesía Joan Fontcuberta

Il me faut préciser que ce n'est pas seulement Butaquita... qui m'a fait penser à ces deux photomontages, mais aussi Tàpia en la calle Toledo, pour une autre raison. Revenons à la genèse des deux images ainsi intitulées... Gómez a placé son Rolleiflex devant le mur, et a pris deux clichés de $6 \times 6 \mathrm{~cm}$, qu'il a ensuite recadrés au tirage, afin d'équilibrer les masses : l'un avec Marichu ; l'autre sans. Pareillement, Fontcuberta a placé son appareil devant le mur, et a pris deux clichés de $6 \times 6 \mathrm{~cm}$, l'un avec la figure (le cycliste ou les deux chiens), l'autre sans. Ensuite, il en a gardé le format, mais a placé successivement les deux images dans son agrandisseur pour réaliser un seul tirage, où l'on voit surgir ces êtres fantastiques dont une partie du corps est en train de s'effacer. La " cuisine " photographique est différente, car on imagine mal Gómez rendant invisible une partie du corps de Marichu, comme le fera quinze ans plus tard le photographe catalan; mais le rapport dialectique entre figure et fond reste semblable pour les deux.

Revenons pour finir aux deux autres images de Paco Gómez que nous avons étudiées, pour y retrouver son jeu sur planéité et profondeur. En 1958-59, dans Cristo, on a vu que celui-ci se réduit aux deux bandes noires. En faisant entrer dans l'image ces franges de hors-champ latéral, il en affirme la présence, sans pour autant en révéler le contenu. Il n'en va pas de même pour Horizontal y vertical, une photo intitulée aussi Medianería ${ }^{34}$, où l'espace ouvert avec les deux lignes des poteaux fait part égale avec l'espace plan du mur. Lourdeur et légèreté, opacité et transparence, obstacle et ouverture... L'un ne va pas sans l'autre dans les séries de photos de murs mitoyens que Gómez désigne par le terme Medianerías. Car pour celui qui pratique depuis 1959 la photographie d'urbanisme et architecture, le mur ne doit pas rester un espace clos, mais interagir avec son environnement. J'en prendrai deux exemples, pour terminer : deux photos madrilènes des années soixante-dix, Medianería con antenas de televisión (Prolongación de la calle 
General Mola), de 1972, et Medianería en la calle General Mola, de 19745. L'élégante composition de découpes de différents murs aveugles dans le cadre presque carré de l'image (format $4 \times 5$ ), aplatis par la frontalité du cadrage, s'ouvre vers le haut avec une forêt d'antennes graciles. La magnifique opacité des différents gris des murs débouche ainsi sur la respiration des toits.

Aujourd'hui, la calle General Mola n'existe plus ; autres temps, autres mœurs, elle est devenue calle del Príncipe de Vergara. Et déjà, en 1974, la jeune génération que l'on a associée à la nouvelle et subversive revue Nueva Lente, trace une autre histoire de la photographie espagnole. Deux membres de cette génération, Joan Fontcuberta et Rafael Levenfeld (tous deux nés en 1955), qui avaient créé en 1981 la revue PhotoVisión, se sont retrouvés pour préparer en 1994 un numéro monographique de cette revue sur Paco Gómez. Cependant - nous explique Levenfeld dans «Francisco Gómez. Notas y recuerdos $»^{36}-$, Joan Fontcuberta venait de recevoir un appel de Marta Gili, directrice alors de la fondation «La Caixa ", lui demandant d'informer son ami que leurs projets allaient être chamboulés. "La Caixa », en effet, venait de programmer la première rétrospective espagnole de Francisco Gómez, et c'est Rafael Levenfeld qui devait en être le commissaire. Celui-ci nous raconte comment cette exposition, qui allait être inaugurée en 1995, a été pour Gómez « el broche de su trayectoria artística » ${ }^{37}$. Trois années plus tard, Francisco Gómez Martínez a rejoint le paradis des photographes ; il avait 80 ans. Sa rétrospective ${ }^{38}$ avait été intitulée La emoción construida; on ne pouvait rêver meilleure expression, pour celui qui savait manier la rigueur de l'architecte et la sensibilité du poète.

\section{BIBLIOGRAPHIE}

Daniel ABADIE (dir.), Tàpies, Paris, Éditions du Jeu de Paume / Réunion des musées nationaux, 1994.

Archivo Paco Gómez. El instante poético y la imagen arquitectónica, Madrid, Comunidad de Madrid / Fundació Fotocolectania, 2016.

Matías BALLESTER CAIRAT, « El ascetismo expresivo de Francisco Gómez », Arte fotográfico, 119 (novembre 1961), p. 972-975.

Francisco Gómez. La emoción construida, Barcelone, Lunwerg / Fundación «la Caixa», 1995.

Leonard KOREN, Wabi-sabi à l'usage des artistes, designers, poètes \& philosophes [1994], Vannes, Éditions Sully - Le Prunier, 2015.

Leonard KOREN, Wabi-sabi : pour aller plus loin [2015], Vannes, Éditions Sully - Le Prunier, 2018.

Rafael LEVENFELD, « Francisco Gómez. Notas y recuerdos », in Archivo Paco Gómez. El instante poético y la imagen arquitectónica, Madrid, Comunidad de Madrid / Fundació Fotocolectania, 2016, p. 327-335.

Alberto MARTín, « La poética de Francisco Gómez. Un itinerario conceptual », in Archivo Paco Gómez. El instante poético y la imagen arquitectónica, Madrid, Comunidad de Madrid / Fundació Fotocolectania, 2016, p. 13-62. 
Ramón MASATS, « Paco Gómez. La sutil elegancia del ladrillo », in Paco Gómez, Madrid, La Fábrica, collection PhotoBolsillo, 2008, non paginé.

Paco Gómez, Madrid, La Fábrica, collection PHotoBolsillo, 2008.

Paco Gómez. Fotografías, Barcelone, RM Editorial / Fundació Fotocolectania, 2010.

Saramaya PELLETEY, Un artiste face au mur : Antoni Tàpies et la poétique de la matière (1955-1975), thèse de doctorat d'études hispaniques, sous la direction de Jacques Terrasa, Aix-Marseille Université, 2012.

Antoni TÀPIES, La pratique de l'art, Paris, Éditions Gallimard, collection « Idées », 1974.

Jacques TERRASA, « Photographie », in Nancy BERTHIER (coord.), Lexique bilingue des arts visuels, Paris, Éditions Ophrys, 2011, p. 117-146.

Jacques TERRASA, Analyse du texte et de l'image en espagnol [1999], Paris, Armand Colin, 2021, 3e éd.

Jacques TERRASA, Joan Fontcuberta / Perfida Imago, Cognac, Le temps qu'il fait, 2006.

Laura TERRÉ, « Orden y desorden », in Paco Gómez. Fotografías, Barcelone, RM Editorial / Fundació

Fotocolectania, 2010, p. 17-30.

Laura TERRÉ, Historia del grupo fotográfico AFAL, 1956/1963, Sevilla, Photovisión, 2006.

\section{NOTES}

1. Pour connaître AFAL, lire l'ouvrage de Laura TERRÉ, Historia del grupo fotográfico AFAL, 1956/1963, Sevilla, Photovisión, 2006.

2. Jacques TERRASA, «Photographie», in Nancy BERTHIER (coord.), Lexique bilingue des arts visuels, Paris, Éditions Ophrys, 2011, p. 119.

3. Alberto martín, «La poética de Francisco Gómez. Un itinerario conceptual », in Archivo Paco Gómez. El instante poético y la imagen arquitectónica, Madrid, Comunidad de Madrid / Fundació Fotocolectania, 2016, p. 17.

4. Jacques TERRASA, Analyse du texte et de l'image en espagnol [1999], Paris, Armand Colin, 2021, $3^{\mathrm{e}}$ éd., p. 217-224.

5. Ibid., p. 217-218.

6. Ibid., p. 224.

7. Rafael LEVENFELD, «Francisco Gómez. Notas y recuerdos", in Archivo Paco Gómez, op. cit., p. 328-329.

8. Sur La Palangana, lire Laura TERRÉ, Historia del grupo fotográfico AFAL, op. cit., p. 194-201.

9. Laura TERRÉ, «Orden y desorden", in Paco Gómez. Fotografías, Barcelone, RM Editorial / Fundació Fotocolectania, 2010, p. 17-30.

10. Le choix éditorial fait en 2015 dans Archivo Paco Gómez, op. cit., est bien plus large. Ce sont des centaines de photographies qui sont reproduites, montrant les différentes facettes de l'œuvre de Paco Gómez : photos d'architecture, portraits, autoportraits, images urbaines...

11. Cité par Alberto MARTín, «La poética de Francisco Gómez. Un itinerario conceptural », in Archivo Paco Gómez, op. cit., p. 29.

12. Ramón MASATS, «Paco Gómez. La sutil elegancia del ladrillo», in Paco Gómez, Madrid, La Fábrica, collection PhotoBolsillo, 2008, non paginé.

13. Alberto MARTín, op. cit., p. 20.

14. Rafael LEVENFELD, op. cit., p 329.

15. Alberto MARTín, op. cit., p. 20. 
16. Fragment de conversation avec le photographe, cité dans Laura TERRÉ, « Orden y desorden », op. cit., p. 27.

17. Ramón MASATS, op. cit., non paginé.

18. Rafael LEVENFELD, op. cit., p. 329.

19. Entretien de 1991, cité dans Laura TERRÉ, « Orden y desorden », op. cit., p. 28.

20. Alberto MARTín, op. cit., p. 21.

21. Matías balleSTER CAIRAT, «El ascetismo expresivo de Francisco Gómez », Arte fotográfico, 119 (novembre 1961), p. 972-975.

22. Alberto Martín a écrit, au sujet de l'article de Matías Ballester : «El análisis tiene una profundidad desacostumbrada para el nivel que ofrecen los textos críticos de la época sobre fotografía, situándose más cerca del ámbito plástico que del fotográfico. » (Alberto MARTín, op. cit. p. 22.)

23. Voir par exemple Ocre-gris, $n^{\circ}$ LXX (1958), Grande peinture (1958) ou Pyramidal (1959), dans Daniel ABADIE (dir.), Tàpies, Paris, Éditions du Jeu de Paume / Réunion des musées nationaux, 1994, respectivement p. 95, 97-98 et 102-103.

24. Dans "Communication sur le mur ", chapitre XII de Antoni TÀPIES, La pratique de l'art, Paris, Éditions Gallimard, collection «Idées », 1974, p. 206-215.

25. Saramaya Pelletey, Un artiste face au mur: Antoni Tàpies et la poétique de la matière (1955-1975), thèse de doctorat d'études hispaniques, sous la direction de Jacques Terrasa, Aix-Marseille Université, 2012.

26. Les termes sont de Laura TERRÉ, « Orden y desorden », op. cit., p. 22.

27. Alberto MARTín, op. cit., p. 36.

28. Id.

29. «Las diez fotografías presentadas tienen formato $30 \mathrm{x} 40$, y entre ellas se reconocen algunas de las más célebres del fotógrafo como Banco en el jardín botánico, Mesa con mantel blanco a la puerta de una casa, Butaquita en un frontón, etc. ", Laura TERRÉ, Historia del grupo fotográfico AFAL, op. cit., p. 160.

30. C'est ce que l'on a pris coutume d'appeler aujourd'hui le wabi-sabi, un concept japonais rendu célèbre par les deux ouvrages de Leonard KOREN, Wabi-sabi à l'usage des artistes, designers, poètes \& philosophes [1994], Vannes, Éditions Sully - Le Prunier, 2015 et Wabi-sabi : pour aller plus loin [2015], Vannes, Éditions Sully - Le Prunier, 2018.

31. Ces deux photographies ont été évidemment prises le même jour, car la disposition des feuilles est identique sur les deux. L'image sans Marichu était intitulée Tapia y árbol et datée de 1962 dans le catalogue de l'exposition Francisco Gómez. La emoción construida (1995), puis Tapia en la calle Toledo (1961) dans le PHotoBolsillo de 2008 (où l'on trouve aussi Tapia en la calle Toledo, con Marichu, 1961), et enfin S/T, 1962, dans Paco Gómez. Fotografías (2010). Elle n'est pas reproduite dans Archivo Paco Gómez (2016), où l'on a par contre l'autre photo, page 343, simplement intitulée Marichu, et datée aussi de 1961. Aussi, nous avons décidé de garder pour les deux photos les titres du PHotoBolsillo, car le choix a été supervisé par Ramón Masats, un proche de Paco Gómez. Et 1961, car l'image avec Marichu ayant été utilisée pour le numéro 32 de la revue $A F A L$, daté de septembre-octobre 1961, la datation ne fait pas de doute.

32. Sur les premières photographies de Fontcuberta, lire le chapitre 1 de Jacques TERRASA, Joan Fontcuberta / Perfida Imago, Cognac, Le temps qu'il fait, 2006, p. 10-27.

33. Ibid., p. 17.

34. Archivo Paco Gómez, op. cit., p. 209.

35. Ibid., p. 208 et 209 , respectivement.

36. Ibid, p. 334.

37. Id. 
38. Catalogue Francisco Gómez. La emoción construida, Barcelone, Lunwerg / Fundación «la Caixa», 1995. La photo Tapia en la calle Toledo avait été choisie pour la couverture.

\section{RÉSUMÉS}

Vers la fin des années 1950, une nouvelle génération de photographes espagnols se réunit autour de l'association et la revue $A F A L$, et propose une vision humaniste et contextualisée de la société de l'époque. À Madrid, six d'entre eux vont créer en 1959 un groupe informel qu'ils nomment «La Palangana ». Parmi eux, Paco Gómez (1918-1998) est le seul à faire des murs de la capitale ou des alentours de Madrid, non pas le décor de portraits en situation, mais au contraire, un sujet à part entière. C'est autour de 1960 qu'il réalisera ses plus beaux clichés, tandis que, parallèlement, il se spécialise dans la photographie d'architecture. À partir d'un choix restreint d'images de murs de cette période, nous verrons comment se dégage une austérité silencieuse et poétique de la rigueur formelle de ces photographies qui nous invitent à méditer sur la beauté éphémère et imparfaite du monde.

Towards the end of the 1950's, a new generation of Spanish photographers gathered around the association and the magazine AFAL, and it offers a humanist and contextualized vision of the society of the time. In Madrid, six of them set up in 1959 an informal group which they called « La Palangana ». Among them, Paco Gómez (1918-1998) is the only one to make of the walls of the capital or the surroundings of Madrid, not the decoration of portraits in situation, but on the contrary, a subject in its own right. It was around 1960 that he took his most beautiful pictures, while at the same time he specialized in architectural photography. From a limited choice of wall images from this period, we will see how a silent and poetic austerity emerges from the formal rigor of these photographs which invite us to meditate on the fleeting and imperfect beauty of the world.

\section{INDEX}

Mots-clés : photographie espagnole, murs, La Palangana, Paco Gómez, Gabriel Cualladó, Joan Fontcuberta

Keywords : spanish photography, walls, La Palangana, Paco Gómez, Gabriel Cualladó, Joan Fontcuberta

\section{AUTEUR \\ JACQUES TERRASA}

Sorbonne Université 